\title{
Minocycline sensitivity related to the phage type of multiply resistant staphylococci
}

\author{
GORDON RICH ${ }^{1}$ AND JUDITH DAVIDSON
}

From the Regional Public Health Laboratory, Newcastle General Hospital, Newcastle upon Tyne

SYNOPSIS Clinical isolates of multiply antibiotic-resistant strains of Staphylococcus aureus were divided into three groups by phage typing. The most prevalent type, which is usually cloxacillin resistant, was found to be moderately sensitive to minocycline. Unfortunately the degree of sensitivity is not sufficient to warrant the use of the antibiotic in severe staphylococcal infection.

Minocycline is an antibiotic recently released for use in the United Kingdom. The molecule has the basic tetracycline configuration with the addition of an extra dimethylamine group at the 7 position. It is claimed to have increased activity against both tetracycline sensitive and tetracycline resistant organisms, and it has been shown (Martell and Boothe, 1967; Mitchell, 1974) that this applies to Staphylococcus aureus.

A large proportion of staphylococcal infection in the Newcastle General Hospital is due to strains which are resistant to cloxacillin, as well as to penicillin, tetracycline, and streptomycin. It was decided some years ago that an antibiotic policy was advisable to manage these infections. Several alternative agents were available; some are difficult to administer or toxic, others are relatively ineffective in staphylococcal infection. Lincomycin and fusidic acid seemed to be effective and yet relatively nontoxic agents but there was the risk that drug resistance to these agents would arise if they were used on their own (Hale and Selkon, 1970). It was therefore decided to recommend that these antibiotics should always be used together.

Recently reports of pseudomembranous colitis being caused by lincomycin have appeared (Scott et al, 1973). Therefore it seemed of interest to assess the antibacterial activity of minocycline against cloxacillin resistant strains of staphylococci to find if it was an acceptable therapeutic alternative to the continued use of lincomycin and fusidic acid.

The predominance of cloxacillin resistant strains in this hospital is due to phage type $75 / 85$ (Selkon

${ }^{1}$ Present address: Institute of Medical and Veterinary Science, Box 14, Rundle Street, P.O., Adelaide, S. Australia 5000.

Received for publication 2 December 1974. and Stokes, 1972). The phage type was determined as well as the susceptibility to antibiotics.

\section{Methods}

The minimal inhibitory concentrations (MIC) of minocycline and tetracycline and phage susceptibility were determined for 58 tetracycline resistant strains of Staph. aureus. These were isolated from 56 patients with a variety of lesions between March and July 1973. One patient with severe burns yielded three different strains of Staph. aureus during this period. The sensitivity of each strain was tested by the disc diffusion method on Oxoid DST agar with $5 \%$ lysed horse blood incubated at $37^{\circ} \mathrm{C}$. The zone diameter was measured and compared with that for the Oxford staphylococcus (NCTC 6571). The antibiotic discs used were penicillin 1 unit, tetracycline $25 \mu \mathrm{g}$, minocycline $10 \mu \mathrm{g}$, streptomycin $25 \mu \mathrm{g}$, chloramphenicol $25 \mu \mathrm{g}$, clindamycin $2 \mu \mathrm{g}$, and fusidic acid $10 \mu \mathrm{g}$. Cloxacillin sensitivity was determined in a similar manner but using methicillin $10 \mu \mathrm{g}$ discs and incubation at $30^{\circ} \mathrm{C}$ (and checked by testing on salt agar plates containing $5 \% \mathrm{wv} \mathrm{NaCl}$ at $37^{\circ} \mathrm{C}$ ).

The MIC of minocycline and tetracycline for each strain was assayed by plating on to a series of DST agar plates containing doubling concentrations of antibiotics from $0.075 \mu \mathrm{g}$ to $20 \mu \mathrm{g}$ per $\mathrm{ml}$. The inoculum was prepared by making a $1 / 100$ dilution of an overnight nutrient broth culture of the test strains and the Oxford staphylococcus. A $5 \mathrm{~mm}$ loopful of this solution was streaked on to each antibiotic plate and a control plate not containing antibiotic.

Eight strains and the Oxford staphylococcus were inoculated on to each plate. The MIC was 


\begin{tabular}{|c|c|c|c|c|c|c|c|}
\hline \multirow[t]{3}{*}{ Susceptibility to Minocycline } & \multirow[t]{3}{*}{$M I C(\mu g / m l)$} & \multicolumn{6}{|c|}{ Susceptibility to Cloxacillin } \\
\hline & & \multicolumn{2}{|c|}{ Phage type $75 / 85$} & \multicolumn{2}{|c|}{ Non-Phage Reactors } & \multicolumn{2}{|c|}{ Other Phage Types } \\
\hline & & Sensitive & Resistant & Sensitive & Resistant & Sensitive & Resistant \\
\hline Resistant & $\begin{array}{r}10 \\
5\end{array}$ & & 1 & $\begin{array}{l}4 \\
6\end{array}$ & & 1 & \\
\hline Moderately sensitive & $\begin{array}{l}2.5 \\
1.25\end{array}$ & $\begin{array}{l}1 \\
3\end{array}$ & $\begin{array}{l}11 \\
17\end{array}$ & $\begin{array}{l}1 \\
2\end{array}$ & $\begin{array}{l}1 \\
1\end{array}$ & 2 & 1 \\
\hline Sensitive & $\begin{array}{l}0.625 \\
0.312\end{array}$ & 1 & & 2 & & $\begin{array}{l}2 \\
1\end{array}$ & \\
\hline Total & & 5 & 29 & 15 & 2 & 6 & 1 \\
\hline
\end{tabular}

Table Phage type and susceptibility to minocycline and cloxacillin of 58 tetracycline resistant staphylococci

taken as the lowest concentration on which there was no visible growth to the naked eye after 18 hours' incubation at $37^{\circ} \mathrm{C}$. All the strains were 'phage' typed at a routine test dilution and $100 \times$ RTD, using the standard staphylococcus phage set available from the Staphylococcus Reference Laboratory, Colindale.

\section{Results}

Forty-one of the 58 strains were lysed by phage at RTD; the remaining 17 strains showed no lysis by phages at either RTD or $100 \times$ RTD. Thirtyfour strains were typed 75/85, 29 being lysed by both 75 and 85 , and five strains by either 75 or 85 alone (these five strains were classified with type 75/85 as they differed by only a single factor). Two isolates were lysed by phages 6 and 75, both had MICs of $0.6 \mu \mathrm{g} / \mathrm{ml}$ of minocycline. Single isolates occurred of phage types 50/81, 84/85, 29/72, 77, and 75/77/84.

All 58 strains of Staph. aureus were sensitive to chloramphenicol, lincomycin, and fusidic acid. Most of these were resistant to streptomycin (52) and all were resistant to penicillin and tetracycline. Three strains had an MIC of $2.5 \mu \mathrm{g} / \mathrm{ml}$ tetracycline, and the other 55 strains were inhibited only by 5 or more $\mu \mathrm{g} / \mathrm{ml}$. The table shows the relationships between phage type and sensitivity to minocycline and cloxacillin. Only one of the $\mathbf{3 4}$ strains of phage type $75 / 85$ was resistant to minocycline compared with 10 of the 17 non-phage reacting strains $(P=0.01)$. Resistance to cloxacillin was present in $32(55 \%)$ of the strains, and 29 of these were lysed by phages 75 and/or 85. One of these strains was resistant to minocycline (MIC $10 \mu \mathrm{g} / \mathrm{ml}$ ) but the other 28 strains were considered moderately sensitive to minocycline, having MICs of $1.25 \mu \mathrm{g} / \mathrm{ml}$ or 2.5 $\mu \mathrm{g} / \mathrm{ml}$. (Of the 12 minocycline resistant strains only two were typable with the phage set used.)

\section{Discussion}

The epidemic strain of multiply resistant Staph. aureus found in this hospital has been shown to be moderately sensitive to this new tetracycline antibiotic. Only one isolate could be considered fully sensitive, having an MIC of $0.625 \mu \mathrm{g} / \mathrm{ml}$ of minocycline, and one was resistant. The other 32 isolates of this type had MICs of 1.25 or $2.5 \mu \mathrm{g} / \mathrm{ml}$. The MICs of these cloxacillin resistant isolates lie very close to the maximum serum levels which are likely to be obtained by the recommended dosage of minocycline (MacDonald et al, 1973). Garrod et al (1973) recommended that the concentration of an antibiotic obtainable in the blood should exceed by several fold the MIC of the infecting organism. Consequently we would hesitate to prescribe this drug for a patient whose life is threatened by a cloxacillin resistant staphylococcus. Moreover it has been shown that the levels in some tissues may exceed the blood levels (MacDonald et al, 1973). It may therefore be possible to use minocycline for less severe tissue infections if a larger dosage was used, or in the treatment of urinary tract infections.

The small number (7) of other phage types of Staph. aureus isolated appear to be heterogeneous in their susceptibility to phage and antibiotic. Most were at least moderately sensitive to minocycline though they were resistant to tetracycline (MIC $\geqslant 5 \mu \mathrm{g} / \mathrm{ml}$ ). Among the 17 strains which did not react with the phages used 10 were resistant to minocycline and were sensitive to cloxacillin. This suggests that they form an homogenous group which unfortunately cannot be delineated by the present standard set of phages.

A relationship between phage type and minocycline sensitivity was reported by Phair and Carleton (1970) when they tested 50 tetracycline resistant strains of Staph. aureus. The 23 strains which gave small zones $(15-18 \mathrm{~mm})$, and presumably had reduced sensitivity, were all phage type $84 / 85$, but only two of the 27 giving larger zones (that is, sensitive strains) were of this type. In the present study it was found that sensitivity to minocycline was related to phage type rather than to sensitivity 
to tetracycline or cloxacillin. It may be some time before the mode of transfer of resistance to minocycline is compared to that for tetracycline but it appears that resistance is not determined by the same genetic material. We agree with the conclusion of Leigh and Simmons (1974) that it is necessary to do routine sensitivity testing to tetracycline and minocycline separately if both antibiotics are in use.

\section{References}

Garrod, L. P., Lambert, H. P., and O'Grady, F. (1973). Antibiotic and Chemotherapy, 4th edition, p. 278. Churchill Livingstone, London.

Hale, J. H. and Selkon, J. B. (1970). Multiply resistant staphylococci. (Letter). Lancet, 2, 528-529.
Leigh, D. A. and Simmons, K. (1974). Effect of minocycline on tetracycline-resistant Staphylococcus aureus. (Letter). Lancet, 1, 1006.

MacDonald, H., Kelly, R. G., Allen, E. S., Noble, J. F., and Kanegis, L. A. (1973). Pharmacokinetic studies on minocycline in man. Clin. Pharmacol. Ther., 14, 5, 852-861.

Martell, M. J. Jr. and Boothe, J. H. (1967). The 6-deoxytetracyclines. VII. Alkylated aminotetracyclines possessing $\mathbb{\Phi}$ unique antibacterial activity. J. med. Chem., 10, 44-46.

Mitchell, A. A. B. (1974). Comparative activity of minocycline and tetracycline. (Letter). Brit. med. J., 1, 576-577. $\rightarrow$

Phair, J. P. and Carleton, J. (1970). Susceptibility of staphylo - . cocci to minocycline in vitro. Infect. Immun., 2, 669671.

Scott, A. J., Nicholson, G. I., and Kerr, A. R. (1973) Lincomycin as a cause of pseudomembranous colitis. Lancet, 2, 1232-1234.

Selkon, J. B. and Stokes, E. R. (1972). Chemotherapy of cloxacillin resistant coagulase positive staphylococci. Mast Symposium, Liverpool. (Unpublished). 